\title{
Acquisition in the Morris swim task is impaired by a benzodiazepine but not an NMDA antagonist: A new procedure for distinguishing acquisition and performance effects
}

\author{
JULIAN R. KEITH and MARK GALIZIO \\ University of North Carolina, Wilmington, North Carolina
}

\begin{abstract}
The problem of learning versus performance continues to challenge researchers interested in drug effects on place learning and memory. To address this problem, the present study adapted the repeated acquisition/performance (RAP) procedure to the Morris swim task. The procedure involved training subjects to swim to a hidden platform that was always in the same location in one pool and in a new location each day in another pool. The advantages of this procedure are that acquisitions of new place responses and performances of previously learned place responses can be directly compared in individual subjects within single sessions, and dose-response functions can be determined for each individual subject. Experiment 1 demonstrated the ability of rats to learn new platform locations in one pool while maintaining stable performance on a previously learned place response in another pool within individual sessions. Experiment 2 used the RAP adaptation of the Morris swim task to study effects of chlordiazepoxide (CDZ), a benzodiazepine, and dizocilpine (DZP; a noncompetitive $N$-methyl$D$-aspartate antagonist), on place learning and performance. Both drugs impaired performance in a dose-dependent manner. More interestingly, a moderate dose $(10 \mathrm{mg} / \mathrm{kg})$ of CDZ caused a small but reliable acquisition impairment without affecting performance of the previously learned place response. Doses of DZP that were substantially greater than doses previously shown to disrupt induction of NMDA-dependent long-term potentiation in the hippocampus did not impair acquisition in this procedure. Acquisition was affected only at DZP doses that also impaired the performance measure.
\end{abstract}

The Morris (1981) swim task is widely used to study drug effects on learning and memory. Two procedures are often used together to distinguish drug effects on learning versus performance variables. One procedure requires subjects to swim in a circular pool to a platform located in a fixed position and submerged just beneath the surface of clouded water (hidden platform task). A second, control, procedure requires subjects to swim to an escape platform that extends above the water surface (visible platform task). The hidden platform component is generally interpreted as a test of spatial learning, whereas the visible platform component serves as a control for nonassociative (e.g., sensorimotor and motivational) effects of drugs on performance.

The authors thank Erin Yard, Carl Schmidt, Carmen McLamb, and Doug Shytle for their assistance collecting data as well as David Ziegler and Raymond Pitts for their helpful comments on a previous version of this manuscript. The authors also acknowledge the valuable comments provided by anonymous reviewers of the manuscript. This research was supported by Grant DA 7380-01 from the National Institute on Drug Abuse (M.G.), a University of North Carolina at Wilmington Faculty Research and Development award (J.R.K.), and a College of Arts and Sciences Summer Initiative award (J.R.K.). The DZP data were reported at the 1991 annual meeting of the Society for Neuroscience in New Orleans. Correspondence should be addressed to J. R. Keith, Department of Psychology, University of North Carolina, Wilmington, NC 284033297 (keithj@pop.uncwil.edu).
The hidden and visible platform procedures are not equally sensitive to drug effects. Specifically, some drugs (e.g., cholinergic antagonists, $N$-methyl-D-aspartate [NMDA] receptor antagonists, opioids, and benzodiazepines [BZDs]) impair performances on the hidden platform task at doses that do not impair visible platform performance (for a review, see McNamara \& Skelton, 1992). Such outcomes are sometimes interpreted as evidence that certain drugs produce place learning impairments that are not accounted for by sensorimotor disturbances (see, e.g., Barnes, 1988; G. S. Robinson, Crooks, Shinkman, \& Gallagher, 1989). Consistent with this view are data from studies that compared drug effects on hidden platform acquisition to effects on hidden platform performance swimming to a previously learned platform location (i.e., a retention test). The salient result from the retention studies was that certain drugs (such as NMDA receptor antagonists and benzodiazepines), when given to rats naive to the Morris swim task, impaired hidden platform acquisition, but when these drugs were given to rats that had received prior training, they failed to impair retention performance (McLamb, Williams, Nanry, Wilson, \& Tilson, 1990; McNamara \& Skelton, 1991; Whishaw \& Auer, 1989).

Because the task demands during the acquisition and retention phases of these studies were equivalent, the impairments noted in subjects drugged prior to acquisition 
training and the spared performance observed in subjects drugged only prior to the retention test apparently strengthened the case that deficits during acquisition were not due to drug effects on performance. However, previous studies of drug effects on swim task acquisition were cast in a different light by procedures that included swim task training prior to studying drug effects on hidden platform acquisition. Briefly, drugs (NMDA antagonists and cholinergic antagonists) that impaired acquisition of the hidden platform task in naive rats did not impair acquisition in rats that were experienced in the Morris swim task in a different spatial environment (Bannerman, Good, Butcher, Ramsay, \& Morris, 1995; Cain, Saucier, Hall, Hargreaves, \& Boon, 1996; Saucier \& Cain, 1995; Saucier, Hargreaves, Boon, Vanderwolf, \& Cain, 1996). On the basis of these results, the authors concluded that NMDA and cholinergic (muscarinic) activity were not essential for spatial learning and that drug-induced sensorimotor disturbances contributed to poor hidden platform acquisition in naive rats (the significance of these findings for the NMDA-learning hypothesis is discussed in greater detail in Experiment 2 and the Discussion section). Although they were an important improvement over previous methods used to study drug effects on place learning, procedures that included a pretraining component prior to the drug study still did not permit a direct comparison of drug effects on acquisition and performance. Taken together, these studies underscore some of the difficulties involved in developing procedures that reliably distinguish learning and performance effects of drugs in the Morris swim task.

A procedure that has been used effectively by behavioral pharmacologists to distinguish effects of drugs on learning and performance in an operant conditioning setting is the multiple-schedule-repeated-acquisition procedure (Thompson \& Moerschbaecher, 1979). This procedure involves teaching subjects patterns of reinforced keypeck or leverpress responses. In the presence of one stimulus, the same pattern is always effective (performance component). In the presence of a different stimulus, the reinforced leverpress pattern changes each session (acquisition component). In this procedure, in a single experimental session, drug effects on a welllearned sequence can be compared directly with the acquisition of a new sequence. Additionally, with this repeated acquisition/performance (RAP) procedure, an entire dose-response function can be determined for each individual subject (with repeated determinations at each dose), thereby reducing problems often encountered in group designs resulting from individual differences in rate of learning and reactivity to drugs. This sort of within-subject design permits highly sensitive assessments of drug effects to be completed using substantially fewer subjects than are required when between-groups designs are used (Cohn \& Paule, 1995; France, Moerschbaecher, \& Woods, 1991; Thompson, 1978; Thompson \& Moerschbaecher, 1979).

A procedure for studying repeated acquisitions over long periods in the Morris swim task was originally intro- duced by Whishaw (1985b). Whishaw trained rats on a hidden platform task for several months, moving the escape platform to a different location each day. By the 7 th day of training ( 16 trials per day), rats were learning new platform locations in a single trial. This was manifest by significant declines in latency to locate the platform and the use of a more direct route to get to the platform on Trial 2 relative to Trial 1 . Once asymptotic performance was achieved, rats' performances remained very stable throughout the experiment (over 90 days), demonstrating rapid acquisition each day. As Whishaw noted, this procedure can be used repeatedly to assess drug effects (or effects of other interventions) on place learning in individual subjects.

Unlike RAP procedures developed for operant research, however, Whishaw's (1985b) design focused exclusively on acquisitions of new hidden platform positions and did not examine retention of a previously learned position. It remains to be determined whether the RAP procedure, in which rats learn new platform positions while maintaining stable performance to a previously learned position within individual sessions, can be adapted to the swim task. If the RAP procedure could be implemented in this way, drug effects on place acquisition and performance could be compared directly.

The first goal of the present study was to assess the feasibility of adapting the RAP design to the Morris swim task. This was accomplished in Experiment 1 by testing each subject in two different pools (one painted black and the other white). For each subject, the escape platform remained in a fixed location in one pool (the performance component) and varied from session to session in the other pool (the acquisition component). Because rats acquired new platform locations without disruptions in performance, the RAP procedure was then used to study drug effects in Experiment 2. Whether or not NMDA-dependent mechanisms are critical for spatial learning remains controversial (Keith \& Rudy, 1990; Morris, Anderson, Lynch, \& Baudry, 1986; Saucier \& Cain, 1995). Therefore a noncompetitive NMDA antagonist, dizocilpine (DZP), was chosen for study with the RAP procedure. Second, a BZD, chlordiazepoxide (CDZ), was chosen for study because prior research has shown that it can impair place learning without blocking the induction of NMDA-dependent long-term potentiation (LTP) (McNamara, de Pape, \& Skelton, 1993).

\section{EXPERIMENT 1}

\section{Method}

\section{Subjects}

Seven Holtzman Sprague-Dawley (mycoplasm-free) male rats were individually housed with ad-lib access to food and water under continuous lighting. They began training at 80-120 days of age. All subjects were experimentally naive prior to this experiment.

\section{Apparatus}

Two circular fiberglass pools with identical dimensions $(1.52 \mathrm{~m}$ in diameter, $30.5 \mathrm{~cm}$ deep) were used. One pool was painted white and the other was painted black. A clear plastic escape platform, $20.3 \mathrm{~cm}$ in diameter, was submerged $2.5 \mathrm{~cm}$ below the surface of the water. The pools were divided into four equally sized quadrants 


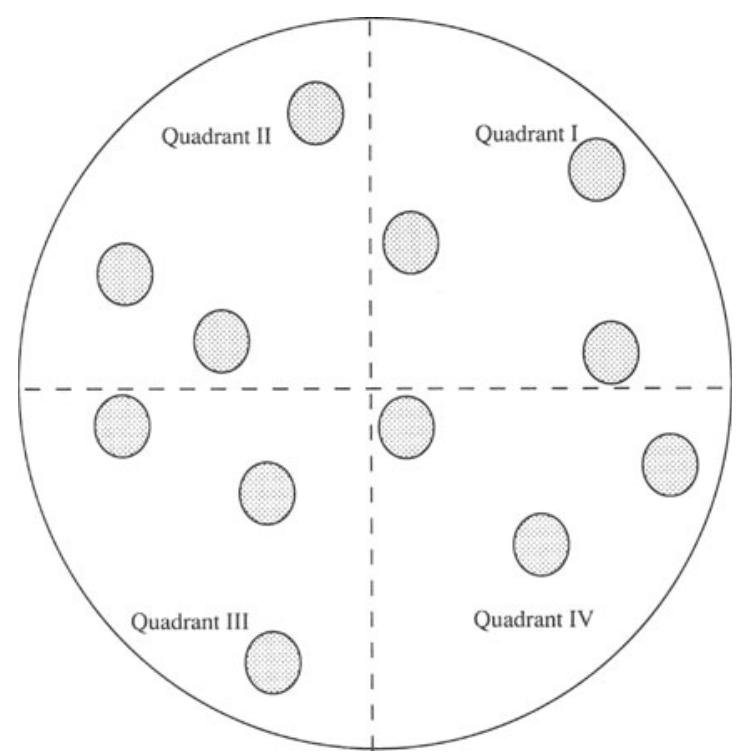

Figure 1. A representation of escape platform positions within the pool used for the acquisition component.

by a string suspended $20 \mathrm{~cm}$ above the water surface. The pools were housed next to one another, $1.5 \mathrm{~m}$ apart, in a $5 \times 6 \mathrm{~m}$ room. The testing room had cinderblock walls and was painted white. Illumination was provided by four pairs of $100 \mathrm{~W}$ fluorescent ceiling fixtures. Water temperature was maintained at $26^{\circ} \mathrm{C}\left( \pm 2^{\circ}\right)$.

The water was clouded by dissolving nontoxic Crayola paint powder in the pools prior to sessions. White paint was used in the white pool and black paint was used in the black pool. Both pools had two black-and-white $(21.5 \times 27.94 \mathrm{~cm})$ cues mounted $15 \mathrm{~cm}$ above and $5 \mathrm{~cm}$ outside the boundaries of the pools. The white pool had one cue that consisted of black horizontal bars $(7.62 \mathrm{~cm}$ in diameter) on the south side and a $4 \times 4$ pattern of black dots $(3.81 \mathrm{~cm}$ in diameter) on the west side. The black pool had a pattern of concentric circles (composed of black lines $7.8 \mathrm{~cm}$ in diameter) on the north side and a cross (composed of black lines $5.08 \mathrm{~cm}$ in diameter). A number of other cues were visible from the pool (e.g., a ventilation fan, etc.). The two experimenters, wearing white lab coats, stood diagonally opposite each other approximately $45 \mathrm{~cm}$ from the pool in the same position each session.

\section{Procedure}

Preliminary training: Navigation training in a single pool. Initially, all rats were trained on the Morris swim task in a single pool. During this phase, 5 rats were trained in the white pool and 2 were trained in the black pool. Each rat received six trials a day with the escape platform in a fixed location. The escape platform remained at the same location throughout training. To begin a trial, a rat was gently placed into the water facing the wall of the pool at one of four starting points and a stopwatch was started. Once a rat stepped onto the platform the stopwatch was stopped. The subject remained on the escape platform for $15 \mathrm{sec}$. If a subject failed to escape by $60 \mathrm{sec}$, it was placed on the platform by the experimenter. Following a trial, the rat was returned to a holding cage where it remained for about $2.5 \mathrm{~min}$. The sequence of start points used was the same for each rat and was changed from day to day. A second dependent variable, quadrant changes, was also measured. As the rat moved about the pool, each time its head passed under one of the strings that marked the quadrant boundaries, a quadrant change was recorded. Training was given 5 days each week (Monday-Friday). Preliminary training continued until subjects met a criterion of three consecutive sessions with escape latencies for each session averaging less than $10 \mathrm{sec}$ per trial.

Repeated acquisition training: Training in the performance and acquisition components. Once subjects met criterion for preliminary training, repeated acquisitions training began. During this phase, rats received six daily training trials on each of two spatial navigation tasks. In the performance component, the submerged platform remained at the same location throughout training and testing. For each rat, the performance task took place in the pool used for that subject during preliminary training. In the acquisition task, the submerged platform was moved to a different location on each day of training and testing. The platform locations used are shown in Figure 1. The sequence of platform positions was randomly determined with the constraint that the same position was not used on consecutive days. The sequence of start points used was the same for each rat and was changed from day to day. For each rat, the performance component took place in the pool used for initial training (i.e., black or white), and the acquisition component took place in the other pool.

For each subject, training trials alternated between the performance and acquisition components. Each session began with a trial in the performance pool, and was followed by a $2.5-\mathrm{min}$ intertrial interval (ITI) and then a trial in the acquisition pool. Subjects received six trials a day in each pool, 5 days a week (Monday-Friday), until they met a 10-session stability criterion. Mean latency for the most recent five sessions was subtracted from the mean latency of the immediately preceding five sessions, and the difference could not exceed $15 \%$ of the 10 -session mean (see Perone, 1991). This criterion had to be met in both the performance and acquisition components. In addition, we required that the subjects average less than $10 \mathrm{sec}$ per trial in the performance task for all six trials and under $10 \mathrm{sec}$ per trial in the acquisition task for Trials 2-6 during the final 10 sessions. The numbers of sessions for each rat in each phase of the experiment are summarized in Table 1 .

\section{Results}

All rats rapidly developed a pattern of swimming directly to the platform in the component where its placement was fixed (performance component). A criterion of three consecutive sessions with all six trials averaging less than $10 \mathrm{sec}$ was required before the acquisition com-

Table 1

Number of Sessions for Each Subject Under Each Training Condition

\begin{tabular}{lcccc} 
& \multicolumn{2}{c}{ Experiment 1 } & & Experiment 2 \\
\cline { 2 - 3 } Subject & $\begin{array}{c}\text { Preliminary } \\
\text { Training }\end{array}$ & $\begin{array}{c}\text { Repeated } \\
\text { Acquisition Training }\end{array}$ & & $\begin{array}{c}\text { Post-Stable } \\
\text { Drug-Free Baseline }\end{array}$ \\
\hline F18 & 6 & 19 & 37 \\
E17 & 4 & 13 & 47 \\
E10 & 4 & 13 & - \\
E16 & 4 & 13 & 37 \\
B13 & 7 & 11 & 48 \\
C3 & 7 & 11 & 45 \\
E22 & 4 & 13 & 26 \\
\hline
\end{tabular}

Note--During preliminary training, subjects received six trials per session in the performance component until they met a criterion of three consecutive sessions with escape latencies for each session averaging less than $10 \mathrm{sec}$ per trial. During repeated acquisition training, subjects received six trials per session in both the performance and acquisition components until they met a 10 -session stability criterion (see text for a description of the stability criterion). In addition, we required that the final 10 sessions average less than $10 \mathrm{sec}$ per trial in the performance component for all six trials and under $10 \mathrm{sec}$ per trial in the acquisition component for Trials $2-6$. 


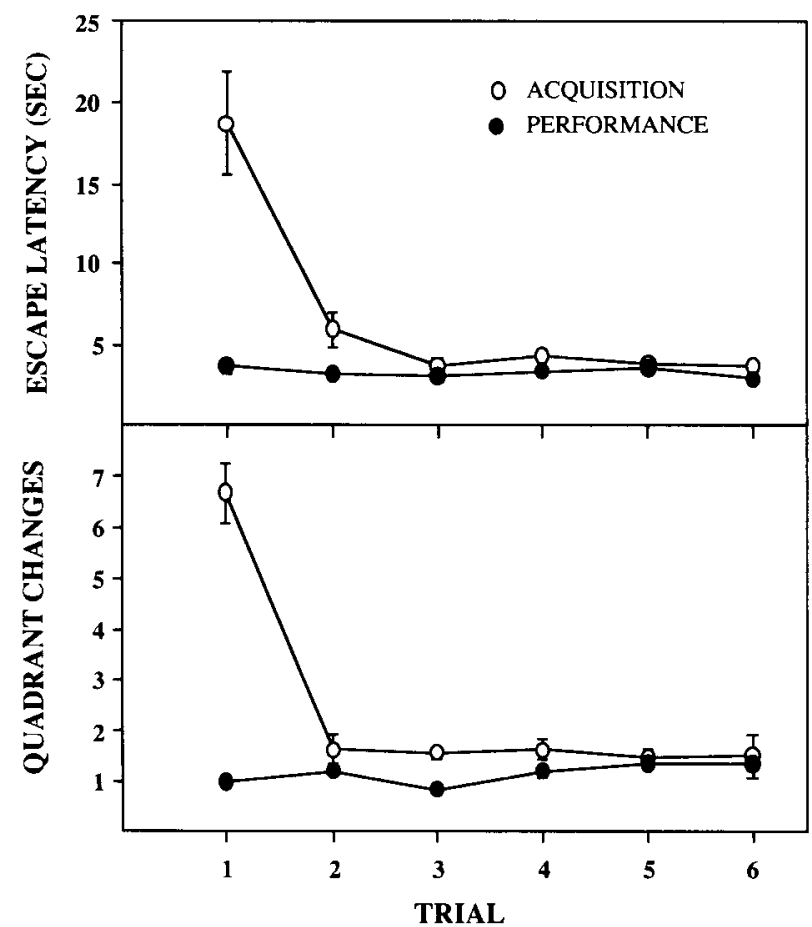

Figure 2. Mean escape latency (top panel) and mean number of quadrant changes (bottom panel) are presented for acquisition (white) and performance (black) components as a function of trials within sessions. The mean of each rat's performance was determined for the last five sessions before the stability criteria were reached. Data presented here represent the means of all 7 rats. Vertical bars indicate standard error of the mean and in cases where no bars are apparent, the bar is concealed by the data point.

ponent was introduced, and this was reached by all rats between the fourth and seventh sessions. The introduction of the acquisition component, in which the platform was in a different place each session, produced only very transient disruptions in behavior. Within a few sessions, all rats had consistently short latencies on trials in the performance component, and longer latencies that declined within individual sessions in the acquisition component. Stable performances in both components developed rapidly, with rats requiring from 11 to 19 sessions to reach the 10 -session criterion. Figure 2 shows mean performances for all rats over the final five training sessions. The top panel shows latency to reach the platform (escape latency) across the trials within the session. Latencies in the performance component (black circles) were short and consistent across trials. In the acquisition component (white circles), latencies were relatively long on the initial trial, as would be expected with a new platform placement, but declined rapidly to levels matching those seen in the performance component. A $2 \times 6$ factorial analysis of variance (ANOVA; within-subjects design) was conducted with task type and trials as the main factors, and it revealed a significant task $\times$ trials interaction $[F(5,30)=16.86, p<.05]$. Parallel results are apparent in the lower panel of Figure 2 for the second de- pendent measure, quadrant changes. The number of maze quadrants entered was high only on the first trial with the new platform placement (acquisition component-white circles), and rapidly declined to minimal levels that were comparable to those observed on all trials in the performance component. The reliability of these data was also confirmed statistically by a significant task $\times$ trials interaction $[F(5,30)=44.78, p<.05]$.

This experiment demonstrated that the RAP procedure is easily adapted to the Morris swim task. The performance task was readily learned, and the introduction of the acquisition component did not disrupt behavior in the performance component. Within 19 or fewer sessions, rats displayed stable, short latencies in the performance component and consistently steep learning curves in the acquisition component. The rapid acquisition of new platform positions observed in the present experiment is consistent with results reported by Whishaw (1985b), the original demonstration of place learning-set formation in the Morris swim task. Our findings are unique, however, in that they demonstrate contextual control of place responding: Rats learned new platform locations in one pool while maintaining stable performance on a previously learned location in another pool.

Because this adaptation of the Morris swim task permits within-session evaluations of both acquisition and performance in individual subjects, it can be used to distinguish selective actions of drugs on place learning versus performance.

\section{EXPERIMENT 2}

The RAP procedure permits the analysis of pharmacological variables that may differentially disrupt performance and acquisition in the Morris swim task. The possibility that one such mechanism, NMDA-mediated LTP in the entorhinal cortex-hippocampus pathway, may be involved in the acquisition of some types of behaviors (e.g., place learning) has been investigated intensively over the past several years (Morris et al., 1986; see Barnes, 1988, for a review).

One approach to the question of whether LTP in this pathway is involved in place learning involves a strategy that combines methods from electrophysiology, pharmacology, and behavior assessment. The logic of the strategy appears straightforward. If a drug blocks the induction of LTP and also produces a place learning impairment (not explained by the drug's effects on sensorimotor or motivational processes), the hypothesis that LTP is a mechanism involved in place learning (but not performance) is supported. The first study to apply this strategy reported that rats treated with an NMDA antagonist, which blocked LTP induction, performed as well as controls on a visible platform discrimination task but were impaired relative to controls on the hidden platform task (Morris et al., 1986).

Later studies developed the analysis of NMDA antagonist effects on place learning even further. For example, Whishaw and Auer (1989) compared the effects of 
DZP, a noncompetitive NMDA antagonist, on three groups of rats and found that DZP impaired acquisition in a group that had to learn new hidden platform positions. Interestingly, the same DZP doses did not impair performance in a group that learned new visible platform positions or a group tested on a well-learned hidden platform position. These results appear to be consistent with the hypothesis that within a certain dose range, DZP selectively disrupted some mechanism unique to the acquisition of new place responses but not mechanisms that support retention, retrieval, and performance of previously learned place, or visible platform responses.

A study of DZP effects on place learning reported by Halliwell and Morris (1987), however, produced results inconsistent with the interpretation given above. In their study, animals given DZP prior to training were impaired on the hidden platform task but were just as good as controls on a retention test (with no additional training given) after the DZP dose was metabolized. This result suggests that DZP masked learning during hidden platform training through effects on general performance.

As mentioned in the introduction, several recent studies further challenged the hypothesis that NMDAdependent mechanisms are essential for place learning in the Morris swim task (Bannerman et al., 1995; Cain et al., 1996; Saucier \& Cain, 1995; Saucier et al., 1996). These studies examined effects of selective NMDA receptor antagonists $\mathrm{D}(-)$-2-amino-5-phosphonovaleric acid (AP5) and NPC17742 (NPC). Unlike the procedures from previous studies of this sort, the behavioral assessment procedures used by Saucier and Cain (1995), Cain et al. (1996), Saucier et al. (1996), and Bannerman et al. (1995) included a group of rats that were familiarized with the swim task via preliminary training in a different room from the one used when drug effects on place learning were later studied. Preliminary training taught rats general procedures necessary for successful swim task performance, such as to swim away from the walls of the apparatus, that there is a submerged platform in the pool, and how to escape by climbing on to the platform. In naive rats, NMDA receptor antagonists produced the expected place learning deficits. In all four studies, however, preliminary training abolished NMDA receptor antagonists effects on place learning in a new environment. These outcomes mean that in pretrained rats, NMDA-dependent mechanisms were not necessary for learning platform locations in a new environment. So, NMDA receptor antagonists (AP5 and NPC) impaired rats' abilities to learn procedural aspects of the Morris swim task, but selective spatial learning impairments were not observed.

The studies discussed above highlighted the importance of including procedural pretraining prior to assessing drug effects on hidden platform acquisition in the Morris swim task. In pretrained rats, NMDA antagonists failed to produce the sort of place learning impairments observed in naive rats (also note that Saucier et al., 1996, found that pretraining eliminated scopolamine-induced impairments). Yet, because none of the studies mentioned above directly compared NMDA antagonist effects on performance and acquisition, it would be premature to conclude that NMDA antagonists' effects on hidden platform acquisition can be explained completely in terms of general effects on performance. It is possible that subtle differential drug effects on acquisition and performance might have been detected if more sensitive procedures were used. The RAP swim task developed for the present study includes extensive pretraining and directly assesses drug effects on procedural performance as well as acquisition of new place responses for each subject within each individual session. Moreover, as seen in Experiment 1 , both performance and acquisition components of the task produced highly stable baselines across sessions. Thus, the RAP procedure should be quite sensitive even to small effects of drugs on either performance or acquisition.

BZD drugs (e.g., CDZ and diazepam), like NMDA antagonists, also impair hidden platform performance at doses that do not impair visible platform performance (Arolfo \& Brioni, 1991; McNamara et al., 1993; McNamara \& Skelton, 1992). Unlike NMDA receptor antagonists, however, $\mathrm{CDZ}$ and diazepam doses that impair place learning do not block induction of LTP in the entorhinalhippocampal pathway (McNamara et al., 1993). Therefore, a comparative analysis of BZD and NMDA receptor antagonist effects on the performance and acquisition components of the Morris swim task should enhance our understanding the pharmacological mechanisms necessary for place learning. In Experiment 2, the effects of $\mathrm{CDZ}$ and DZP on place learning were studied using the RAP adaptation of the Morris swim task.

\section{Method}

\section{Subjects}

Subjects were 6 of the same rats used in Experiment 1 .

\section{Apparatus and Procedure}

The swimming pools used were the same as those described in Experiment 1, and the navigation testing procedures were identical to those used during repeated acquisitions training in Experiment 1 . For humane reasons, when animals were unable to swim under high drug doses, they were immediately removed from the pool and the session was terminated.

\section{Drug Preparation and Administration}

DZP (Research Biochemicals Inc., Natick, MA) and CDZ (Sigma, St. Louis) were dissolved in isotonic $(0.9 \%)$ sodium chloride solution, and intraperitoneal injections were administered in a volume of $1 \mathrm{ml} / \mathrm{kg}$. Drug solutions were prepared fresh daily; doses are expressed in terms of the total salt. DZP doses studied were 0.05 , $0.075,0.1,0.2$, and $0.3 \mathrm{mg} / \mathrm{kg}$. The lower two doses proved ineffective in the first 2 animals studied (C3 and $\mathrm{B} 13)$ and were not tested in the other 4 animals. CDZ doses studied were 3,10 , and $30 \mathrm{mg} / \mathrm{kg}$. Drug and saline injections were administered twice per week (Tuesday and Friday), and sessions were conducted under baseline conditions 3 days per week (Monday, Wednesday, and Thursday). On drug days, subjects received intraperitoneal injections $30 \mathrm{~min}$ (DZP) or $15 \mathrm{~min}(\mathrm{CDZ})$ before the session. DZP was studied in all 6 rats, and CDZ was studied in 3 (B13, C3, and E22). 
Table 2

Number of Sessions Terminated (First Number) and Separate Determinations (Second Number) at Each Dose for Each Rat

\begin{tabular}{lcccc}
\hline & \multicolumn{4}{c}{ Dizocilpine Dose $(\mathrm{mg} / \mathrm{kg})$} \\
\cline { 2 - 5 } \multicolumn{1}{c}{ Subject } & Saline & 0.1 & 0.2 & 0.3 \\
\hline E16 & $0 / 6$ & $1 / 5$ & $1 / 4$ & $1 / 4$ \\
E22 & $0 / 4$ & $0 / 5$ & $1 / 5$ & $1 / 5$ \\
B13 & $0 / 3$ & $0 / 5$ & $1 / 7$ & $1 / 5$ \\
F18 & $0 / 4$ & $0 / 4$ & $0 / 4$ & $2 / 4$ \\
C3 & $0 / 3$ & $0 / 5$ & $1 / 7$ & $1 / 5$ \\
E17 & $0 / 3$ & $0 / 5$ & $2 / 4$ & $1 / 5$ \\
Total & $0 / 23$ & $1 / 24$ & $6 / 31$ & $7 / 28$ \\
Percent terminated & 0 & 4.16 & 19.3 & 25 \\
\hline
\end{tabular}

Table 3

Number of Separate Determinations at Each Dose for Each Rat

\begin{tabular}{lcccr} 
& \multicolumn{4}{c}{ Chlordiazepoxide Dose $(\mathrm{mg} / \mathrm{kg})$} \\
\cline { 2 - 5 } Subject & Saline & 3 & 10 & 30 \\
\hline E22 & 4 & 3 & 3 & 3 \\
B13 & 4 & 3 & 3 & 4 \\
C3 & 4 & 3 & 3 & 4 \\
Total & 12 & 9 & 9 & 10 \\
\hline
\end{tabular}

For rats $\mathrm{B} 13$ and $\mathrm{C} 3$, the $\mathrm{CDZ}$ regimen was completed prior to beginning the DZP regimen. Rat E22 completed the DZP regimen before beginning the $\mathrm{CDZ}$ regimen. Rats studied under both $\mathrm{CDZ}$ and DZP received a minimum five baseline training sessions between drug regimens. Generally, the schedule of drug conditions was randomly generated with the constraint that no dose was administered on successive drug days, and that the end of each cycle (one exposure to each dose including a saline injection) of the drug regimen was completed before beginning the next cycle. Between three and eight separate determinations were made at each dose for each rat. Tables 2 (DZP) and 3 (CDZ) show the number of separate determinations made for each rat at each dose and indicate the number of sessions that were terminated due to inability to swim.

\section{RESULTS}

Figure 3 shows the mean performances for all rats over all baseline (i.e., drug-free) days. These results are shown for two reasons. First, they replicate those presented in Experiment 1, and, second, they indicate that stable patterns of behavior were maintained by each rat on both the acquisition and performance components on the baseline days throughout the drug studies. As is typical in within-subjects behavioral pharmacology studies, baseline performances were maintained following the initiation of drug sessions. This high degree of stability during baseline testing permits a sensitive analysis of subtle effects of drugs on performance and acquisition.

Dizocilpine sometimes produced effects that rendered subjects untestable in the swimming pool. When subjects were unable to swim, the session was immediately aborted and the subject was returned to its home cage. In cases in which sessions were aborted, $60-\mathrm{sec}$ latencies were assigned for each incomplete trial. Table 2 shows the number of aborted sessions recorded for each subject at each DZP dose. The .1-mg/kg DZP dose produced only 1 aborted session (out of 29 total sessions using that dose). The .2- and .3- $\mathrm{mg} / \mathrm{kg}$ doses produced 6 (out of 31 ) and 7 (out of 28) aborted sessions, respectively. CDZ produced no aborted sessions.

Figure 4 shows the effects of DZP on escape latency. The figure shows the mean latencies on each trial in the acquisition (white circles) and performance (black circles) components. DZP increased latencies in a dosedependent fashion in the acquisition component with comparable impairments observed in the performance component as well. This conclusion was confirmed statistically with a within-subjects dose $\times$ task (performance vs. acquisition) $\times$ trials ANOVA that revealed a significant main effect for DZP dose $[F(3,15)=9.41, p<.001]$ and task $[F(1,5)=96.38, p<.001]$, but no significant dose $\times$ task or dose $\times$ task $\times$ trials interactions $(F<$ 1.0). Closely parallel with the latency results were the data for quadrant changes, which are summarized in Table 4. Here the effects of DZP are manifest by increased number of quadrant changes, and, as with the latency data, effects on acquisition accuracy were only apparent at doses that also impaired responding in the performance component.

Individual subject data are presented in Figure 5 with results from the acquisition component in the top panel and the performance component in the bottom panel. Be-

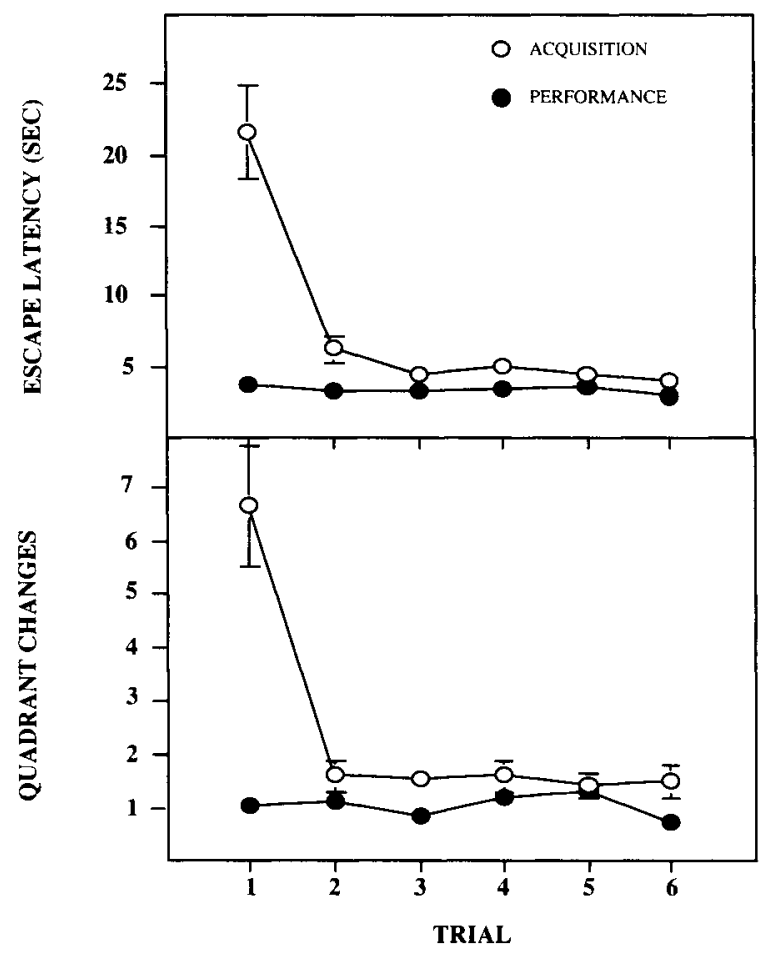

Figure 3. Mean escape latency (top panel) and mean number of quadrant changes (bottom panel) are presented for acquisition (white) and performance (black) components as a function of trials within sessions. The mean of each rat's performance was determined for all baseline sessions conducted during Experiment 2. Data presented here represent the means of the 6 rats. 


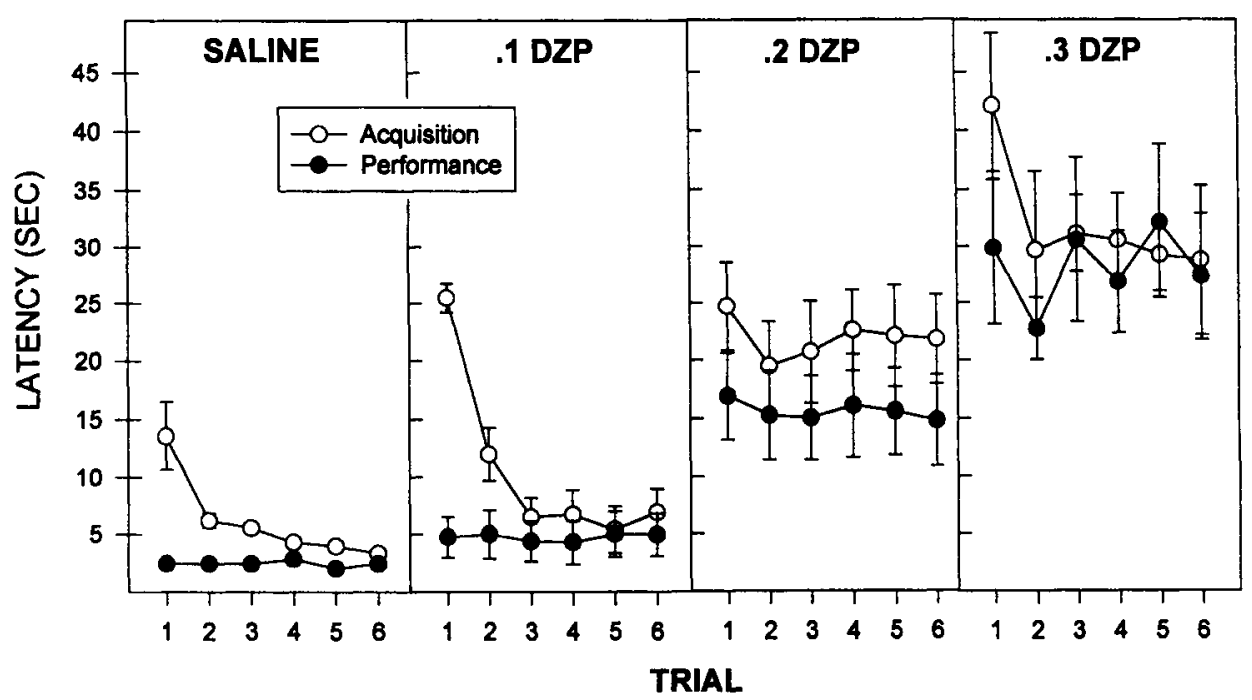

Figure 4. Mean escape latency for acquisition (white) and performance (black) components for each dizocilpine dose as a function of trials within sessions. Data presented here represent the means of the 6 rats. Vertical bars indicate standard error of the mean, and in cases where no bars are apparent, the bar is concealed by the data point.

cause rats' escape latencies reached asymptotic levels by the fourth trial consistently during baseline testing, only the latencies of Trials $2-4$ were used to calculate the means shown in the figure. Each rat's performance represents the mean of all replications at that dose, and is indicated by a different symbol. Although individual differences in sensitivity to DZP are apparent, the overall pattern was quite consistent across subjects. Rat $E 16$ was most sensitive to DZP at every dose and was the only animal that showed clear impairment at the $.1-\mathrm{mg} / \mathrm{kg}$ dose. Most of the rats showed small increases in latency at the $.2-\mathrm{mg} / \mathrm{kg}$ dose and much larger effects at the $.3-\mathrm{mg} / \mathrm{kg}$ dose. Importantly, the effects of DZP on latency in the acquisition component closely paralleled the effects in the performance component.

The dose-response profile for CDZ was quite different from that seen with DZP. There was evidence of selective action of $\mathrm{CDZ}$ on acquisition. Figure 6 shows the effects of $C D Z$ on escape latency. The figure shows the mean latencies for all 3 rats in the acquisition (white circles) and performance (black circles) components. Performance latencies were unchanged, relative to saline sessions, under the $3-$ and $10-\mathrm{mg} / \mathrm{kg} \mathrm{CDZ}$ doses. Under the $3-\mathrm{mg} / \mathrm{kg}$ dose, latencies were longer on Trial 4 , and under the $10-\mathrm{mg} / \mathrm{kg}$ dose, latencies were longer on Trials 2, 3, and 4 relative to the saline sessions. At the 30 $\mathrm{mg} / \mathrm{kg}$ dose, impairments were observed on both components. This conclusion was confirmed statistically with a within-subjects dose $\times$ task (performance vs. acquisition) $\times$ trials ANOVA that revealed a significant main effect for $\operatorname{CDZ}$ dose $[F(3,6)=5.55, p<.05]$ and task $[F(1,2)=17.67, p=.05]$ and a significant dose $\times$ task $\times$ trials interaction $[F(15,30)=3.32, p<.01]$. Post hoc planned comparisons revealed that acquisition latencies under saline differed significantly from latencies under the $10-\mathrm{mg} / \mathrm{kg} \mathrm{CDZ}$ dose on Trials 2 and 4 (and approached significance $[p=.1]$ on Trial 3) and on all six trials under the $30-\mathrm{mg} / \mathrm{kg} \mathrm{CDZ}$ dose. Performance laten-

Table 4

Mean Quadrant Changes During Acquisition (Trials 2-6) and Performance Components Under Each Dizocilpine (DZP) Dose for Each Subject

\begin{tabular}{lccccccccc}
\hline & \multicolumn{9}{c}{ Dose $(\mathrm{mg} / \mathrm{kg})$} \\
\cline { 2 - 8 } & \multicolumn{3}{c}{ Acquisition } & & \multicolumn{3}{c}{ Performance } \\
\cline { 2 - 9 } Subject & Saline & 0.1 & 0.2 & 0.3 & & Saline & 0.1 & 0.2 & 0.3 \\
\hline B13 & 1.86 & 2.08 & 2.56 & 3.20 & & 0.94 & 1.03 & 1.47 & 1.55 \\
C3 & 2.46 & 2.44 & 3.53 & 3.99 & & 0.99 & 1.43 & 1.41 & 1.55 \\
E16 & 1.90 & 1.45 & 5.40 & 4.40 & & 0.63 & 1.00 & 1.33 & 4.33 \\
E17 & 1.13 & 1.45 & 1.19 & 3.05 & 1.16 & 0.87 & 0.83 & 1.33 \\
E22 & 1.45 & 1.33 & 1.46 & 4.66 & & 0.87 & 1.39 & 1.54 & 2.87 \\
F18 & 1.86 & 3.50 & 3.06 & 5.60 & & 0.75 & 0.67 & 1.00 & 4.17 \\
Average & 1.78 & 2.04 & 2.86 & 4.15 & & 0.89 & 1.07 & 1.26 & 2.63 \\
\hline
\end{tabular}

Note-The means shown in this table do not include trials or sessions that were terminated due to DZP-induced impairments in swimming ability. 


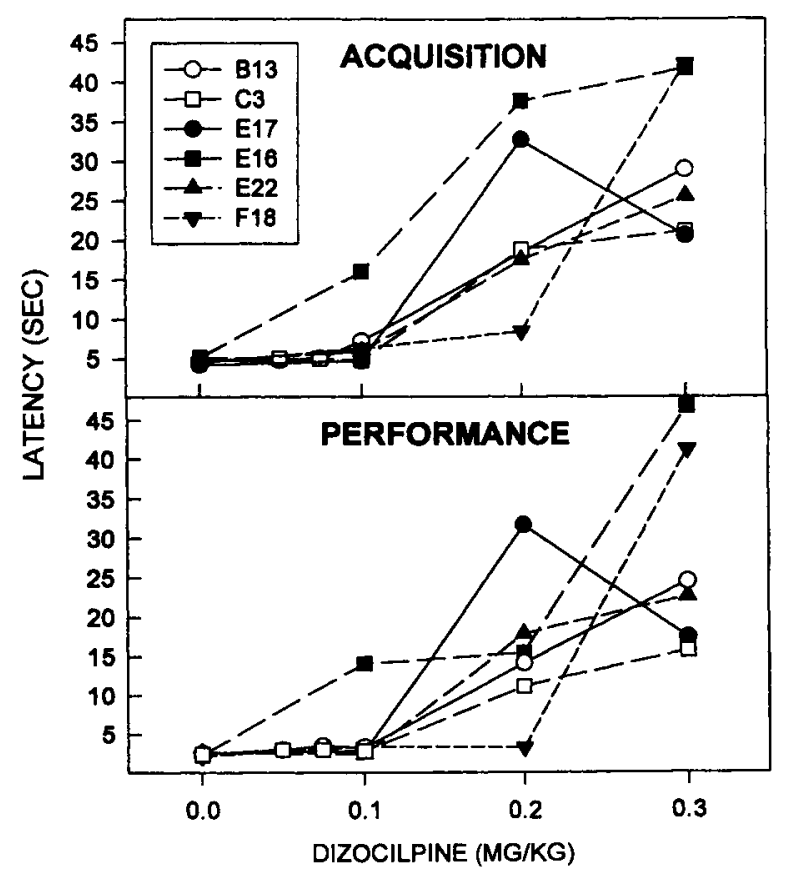

Figure 5. Individual subject data for Experiment 2 are plotted as a function of dizocilpine dose for the acquisition (top panel) and performance (bottom panel) components. Data presented show means of Trials $2-4$.

cies on Trials 1 and 2 under the $30-\mathrm{mg} / \mathrm{kg} \mathrm{CDZ}$ dose were significantly different from the corresponding trials under saline.

Figure 7 presents results from individual subjects. The acquisition latencies are shown in the top panel, and the performance latencies are shown in the bottom panel. Only the latencies of Trials 2-4 were used to calculate the means shown in this figure. Each rat's performance represents the mean of all replications at that dose and is indicated by a different symbol. Neither the 3- nor the $10-\mathrm{mg} / \mathrm{kg}$ dose affected performance latencies in any of the rats. Latencies increased for all 3 rats, however, in the acquisition component under the $10-\mathrm{mg} / \mathrm{kg}$ dose. The $30-$ $\mathrm{mg} / \mathrm{kg}$ dose impaired both components. Again, closely parallel with the latency results were the data for quadrant changes, which are summarized in Table 5. Here the effects of CDZ are manifest by increased number of quadrant changes, and as with the latency data, selective ef- fects on acquisition accuracy were observed at the 10 $\mathrm{mg} / \mathrm{kg}$ dose, a dose that did not impair responding in the performance component.

\section{DISCUSSION}

The present study demonstrated the feasibility and utility of extending the RAP procedure to the analysis of place learning in the Morris swim task. In Experiment 1, within 17-25 training sessions all rats learned to maintain stable, short escape latencies in the performance component and consistently displayed steep learning curves in the acquisition component. These results join with those of previous studies demonstrating that once trained, rats are able to learn new escape platform positions very rapidly (Keith \& McVety, 1988; Whishaw, $1985 \mathrm{~b}$ ). The present study went further than previous studies, however, by examining acquisition and performance in individual subjects within individual sessions.

In Experiment 2, the RAP adaptation of the Morris swim task was used to study the effects of $C D Z$, a benzodiazepine, and DZP, an NMDA antagonist, on place learning and performance. Complete dose-response functions with multiple determinations at each drug dose were generated from individual subjects. Drug effects on place navigation were quite consistent across subjects.

CDZ impaired both components of the RAP swim task in a dose-dependent manner. Importantly, however, an intermediate CDZ dose $(10 \mathrm{mg} / \mathrm{kg})$ impaired acquisition without affecting the performance component. This result further confirms the previous literature on BZD effects on learning and memory, the common finding being that BZDs impair acquisition of new behaviors but not performance of established behaviors (see, e.g., Bickel, Higgins, \& Hughes, 1991). As for the case of BZD effects on place learning in the Morris swim task, diazepam (McNamara \& Skelton, 1991) and CDZ (McNamara \& Skelton, 1992; McNaughton \& Morris, 1987) both have been found to impair hidden platform performance at doses that spared visible platform performance. Furthermore, McNamara and Skelton (1991) reported that a diazepam dose that impaired rats' initial acquisition of a hidden platform position failed to impair a different group's performance swimming to a previously learned position. Our results complement those reported by others (McNamara \& Skelton, 1991, 1992; McNaughton \& Morris, 1987) by revealing selective CDZ-induced acqui-

Table 5

Mean Quadrant Changes During Acquisition (Trials 2-6) and Performance Components Under Each Chlordiazepoxide Dose for Each Subject

\begin{tabular}{lccccccccc}
\multicolumn{8}{c}{ Components Under Each Chlordiazepoxide Dose for Each Subject } \\
\cline { 2 - 10 } & \multicolumn{3}{c}{ Acquisition } & \multicolumn{3}{c}{ Dose $(\mathrm{mg} / \mathrm{kg})$} \\
\cline { 2 - 10 } Subject & Saline & 3 & 10 & 30 & & \multicolumn{3}{c}{ Performance } \\
\hline B13 & 1.80 & 1.33 & 2.87 & 4.75 & 1.04 & 0.94 & 1.06 & 1.88 \\
C3 & 2.10 & 3.05 & 2.25 & 5.55 & 1.08 & 0.94 & 1.06 & 1.67 \\
E22 & 1.25 & 2.07 & 2.07 & 2.80 & 0.67 & 0.78 & 0.89 & 2.22 \\
Average & 1.71 & 2.15 & 2.40 & 4.37 & 0.93 & 0.89 & 1.00 & 1.92 \\
\hline
\end{tabular}




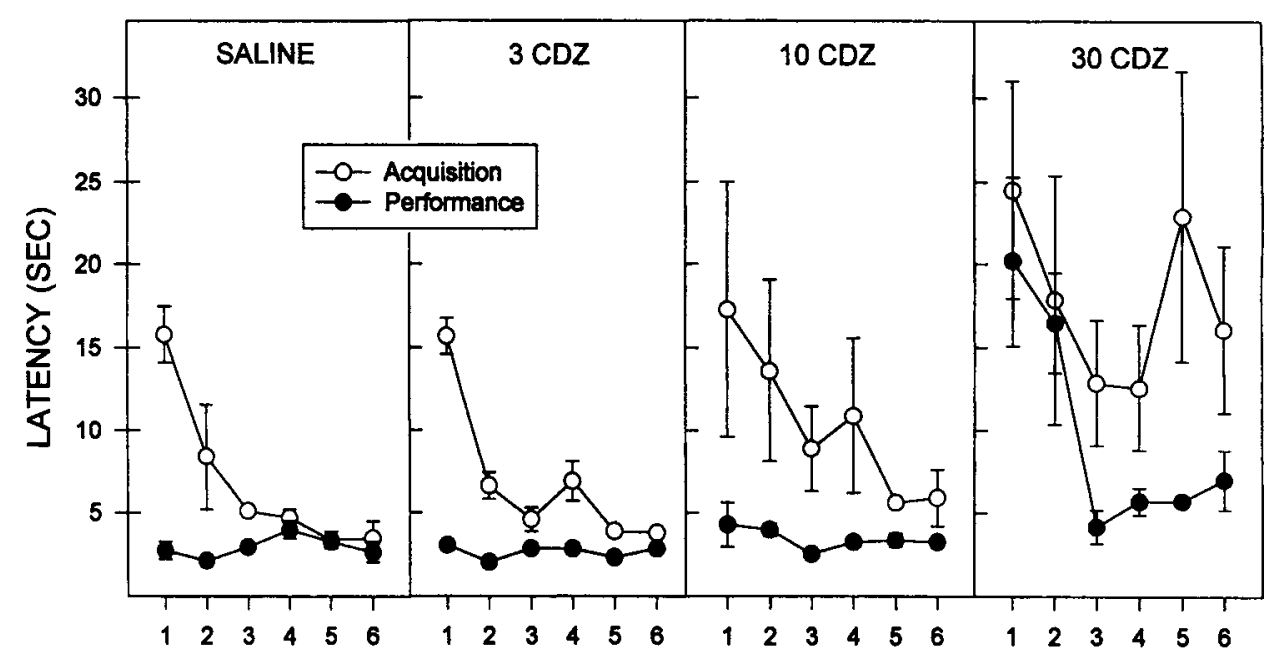

TRIAL

Figure 6. Mean escape latency for acquisition (white) and performance (black) components for each chlordiazepoxide dose as a function of trials within sessions. Data presented here represent the means of the 3 rats. Vertical bars indicate standard error of the mean, and in cases where no bars are apparent, the bar is concealed by the data point.

sition impairments in individual subjects while the same subjects continued to maintain stable baseline-level latencies (and quadrant changes) in the performance component. The CDZ effects observed in this study cannot be explained in terms of general sedation, CDZ effects on swim speed, hypothermia, or state-dependent learning because such effects would be manifest in both the performance and acquisition components of the swim task.

The physiological mechanisms that mediate the effect of $\mathrm{CDZ}$ on place learning are not yet completely understood. As discussed in the introduction to Experiment 2, McNamara et al. (1993) found that neither CDZ nor diazepam suppressed LTP in the entorhinal-hippocampal pathway. There is evidence that systemic BZD/GABA $\mathrm{A}$ agonists reduce $\mathrm{ACh}$ activity in the hippocampus and other forebrain regions (Miller \& Richter, 1985; Richter, Gormley, Holtman, \& Simon, 1982). Manipulations that suppress hippocampal ACh activity (e.g., ACh [muscarinic] receptor antagonists, medial septal lesions) impair hidden platform learning in the Morris swim task (Hagen, Salamone, Simpson, Iversen, \& Morris, 1988; Whishaw, 1985a). It will be valuable in future studies to evaluate $\mathrm{ACh}$ involvement in the RAP adaptation of the Morris swim task.

DZP impaired navigation on the performance component in a dose-dependent manner. However, impaired navigation on the acquisition component was observed only at doses that also impaired performance. Thus, like other recent studies of NMDA antagonist effects on place learning, the results of the present study contradict the hypothesis that NMDA antagonists selectively suppress mechanisms involved in acquisition (Bannerman et al., 1995; Cain et al., 1996; Saucier \& Cain, 1995; Saucier et al., 1996). The failure of DZP to selectively impair acquisition occurred at doses that reliably disrupt
LTP induction (Gilbert \& Mack, 1990; Leung \& Shen, 1993; G. B. Robinson \& Reed, 1992), casting further doubt on the hypothesis that NMDA-dependent LTP is required for spatial learning.

Our analysis of DZP effects on place learning appears to be inconsistent with Whishaw and Auer's (1989) find-

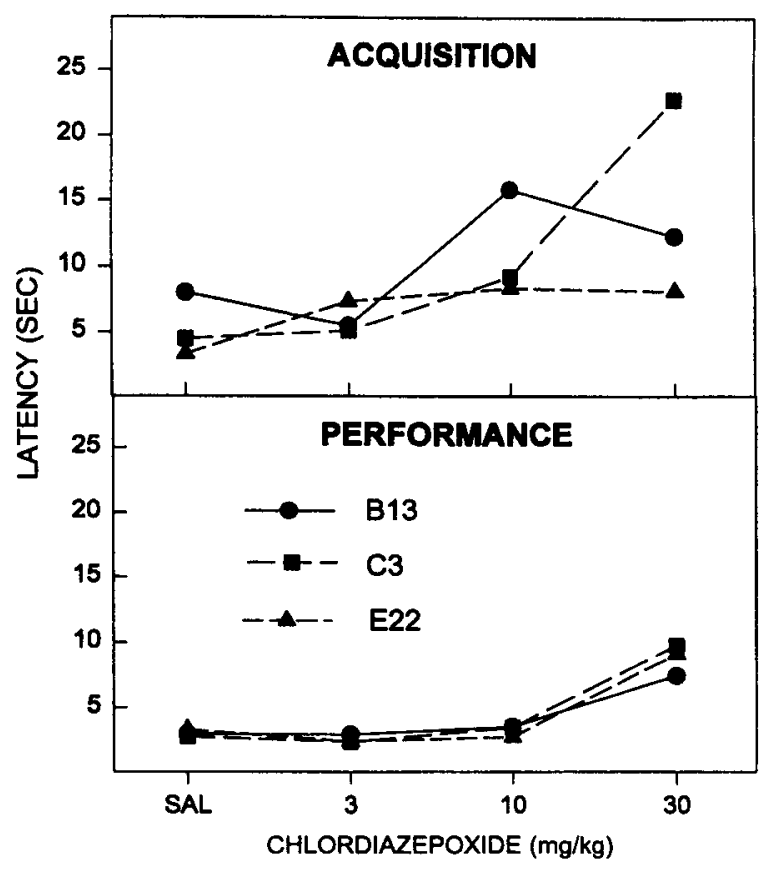

Figure 7. Individual subject data for Experiment 2 are plotted as a function of chlordiazepoxide dose for the acquisition (top panel) and performance (bottom panel) components. Data presented show means of Trials 2-4. 
ings. They found that DZP doses impaired navigation in rats required to learn new place responses but not in groups required to swim to previously learned place or new visible platform positions. However, in the present study prior to initiating drug testing, rats received more than 360 place navigation trials distributed over about 30 days prior to the initiation of drug testing. This degree of pretraining apparently alters the DZP dose-response curve substantially. For instance, other studies that used rats naive to the Morris swim task found that a 0.05 $\mathrm{mg} / \mathrm{kg}$ DZP dose impaired hidden platform learning and a $0.08-\mathrm{mg} / \mathrm{kg}$ dose even impaired visible platform performance (G. S. Robinson et al., 1989). By comparison, in our study a $0.1-\mathrm{mg} / \mathrm{kg}$ DZP dose (which produced notable behavioral abnormalities such as head weaving and imbalance in the home cage immediately prior to testing) failed to impair either performance or acquisition in 5 out of 6 subjects. So, the extensive training given prior to initiating drug testing apparently protected animals against nonassociative performance deficits typically produced by DZP (i.e., wobbly swimming and thigmotaxis). Perhaps DZP effects on swim task performance interact with the catecholamine response to water immersion. Water immersion elevates plasma catecholamines markedly in naive rats, a response that habituates over the course of several weeks in rats that experience daily immersions (Konarska, Stewart, \& McCarty, 1990; Mabry, Gold, \& McCarty, 1995). By this account, repeated training on the Morris swim task may result in habituation of the catecholamine response to water immersion, thus permitting subjects to adapt effectively to the stress associated with NMDA antagonist administration.

Other accounts of the present data could be developed. For example, Jackson, Koek, and Colpaert (1992) recently presented data supporting the notion that NMDA antagonists do not produce deficits in memory or retention, but rather produce strong state-dependent effects. From this perspective, the present results should have been expected because with repeated testing at each dose of DZP, rats would come to learn how to perform in the presence of the DZP cue.

Yet another account is based on a study by Shapiro and O'Connor (1992) that examined DZP effects on radial arm maze performance. They found that DZP (a $0.0625-\mathrm{mg}$ / $\mathrm{kg}$ dose) impaired working-memory-based performance when the radial arm maze was located in a new environment but not when it was located in a familiar environment. The authors proposed that DZP may have produced this effect by disrupting the formation of new spatial representations while not affecting behaviors based on already established representations. The results of our study could be interpreted as being consistent with Shapiro and O'Connor's hypothesis. In our study, place response learning and performance both were studied in environments that were familiar to the subjects. Therefore, in Shapiro and O'Connor's terms, rats learned and performed place responses on the basis of previously learned representations of the testing envi- ronment. Perhaps if the acquisition component of the RAP adaptation of the Morris swim task were carried out in an unfamiliar environment (e.g., novel background stimuli), doses of DZP that selectively impair acquisition would be found.

It should be noted that although Shapiro and O'Connor's (1992) hypothesis is consistent with the pattern of data found in our study, it is difficult to square it with the results reported by Whishaw and Auer (1989). As noted, Whishaw and Auer found doses of DZP that impaired new place response learning in the Morris swim task but not performance of a previously learned place response or a new cued response. In Whishaw and Auer's study, all rats were familiarized with the testing environment during a 10-day, pretraining phase with 16 trials per day prior to initiating drug testing. So, Whishaw and Auer's rats were highly familiar with the testing environment, and yet some doses of DZP still selectively impaired the acquisition of new place responses, a result that directly contradicts Shapiro and O'Connor's hypothesis. Similarly, it is difficult to reconcile the results of the studies by Saucier and Cain (1995), Cain et al. (1996), Saucier et al. (1996), and Bannerman et al. (1995) with Shapiro and O'Connor's hypothesis. In each of these studies, NMDA antagonists failed to impair place learning in rats pretrained in a different environment from the one used during drug testing. While under the influence of an NMDA antagonist, pretrained rats learned enough about a new environment to perform the hidden platform task as well as control rats.

Readers accustomed to the standard Morris swim task protocol may be concerned about the absence of probe trials in the RAP procedure developed for the present study. On probe trials (i.e., transfer testing; Morris, 1981), the platform is removed from the pool and rats' paths and quadrant dwell times are recorded for a fixed period (usually $60-120 \mathrm{sec}$ ) while subjects search for the missing platform. A probe trial is typically used once acquisition training is completed and provides useful data about how well subjects learned the platform location. For instance, subjects that spend a disproportionate amount of time in the quadrant that contained the escape platform during acquisition training learned the platform location better than subjects whose quadrant search times were equally distributed. Such probe testing is a valuable aspect of the standard Morris swim task procedure because latency improvements during acquisition may reflect either learning about the platform location or an effective nonspatial strategy (e.g., swimming in a circle $12 \mathrm{~cm}$ from the pool wall). Although probe trials could easily be incorporated in future studies, the RAP procedure used in the present study circumvented the need for them in several ways. First, under most Morris swim task protocols, the escape platform remains a fixed distance from the pool wall throughout acquisition training, potentially reinforcing a reliable nonspatial search strategy. In contrast, under the RAP procedure used in the present study, each day when the platform was relocated in the 
acquisition pool, it was a different distance from the wall (Figure 1), rendering a nonspatial strategy ineffective. Further evidence that rats in the present study did not use a nonspatial strategy in the acquisition component comes from the acquisition data shown in Figures 2, 3, and 4. Escape latencies on Trial 1 were substantially longer than latencies on the remaining five trials. If rats had a nonspatial strategy that enabled them to quickly find the escape platform, why didn't they use it on the first trial? The decrease in escape latencies and quadrant changes within each session on the acquisition component most likely reflects learning about the new platform locations.

When Whishaw (1985b) first described a procedure for studying repeated acquisitions of place responses using the Morris swim task, he proposed a number of potential uses for such a procedure, including studies of recovery of function from brain damage, brain grafts, drug effects, aging, and environmental enrichment. The RAP adaptation of the Morris swim task presented here could be applied to the same sorts of research questions. Moreover, this new procedure offers the additional benefit of allowing assessments of both acquisition and performance in individual subjects over long periods.

\section{REFERENCES}

Arolfo, M. P., \& Brioni, J. D. (1991). Diazepam impairs place learning in the Morris water maze. Behavioral \& Neural Biology, 55, 131-136.

Bannerman, D. M., Good, M. A., Butcher, S. P., Ramsay, M., \& MorRis, R. G. M. (1995). Distinct components of spatial learning revealed by prior training and NMDA receptor blockade. Nature, $\mathbf{3 7 8}$, 182-186.

BARNES, C. A. (1988). Spatial learning and memory processes: The search for their neurobiological mechanisms in the rat. Trends in Neurosciences, 11, 163-169.

Bickel, W. K., Higgins, S. T., \& Hughes, J. R. (1991). The effects of diazepam and triazolam on repeated acquisition and performance of response sequences with an observing response. Journal of the Experimental Analysis of Behavior, 56, 217-237.

Cain, D. P., Saucier, D., Hall, J., Hargreaves, E. L., \& Boon, F. (1996). Detailed behavioral analysis of water maze acquisition under APV or CNQX: Contribution of sensorimotor disturbances to druginduced acquisition deficits. Behavioral Neuroscience, 110, 86-102.

Cohn, J., \& PAULe, M. G. (1995). Repeated acquisition of response sequences: The analysis of behavior in transition. Neuroscience \& Biobehavioral Reviews, 19, 397-406.

France, C. P., Moerschbaecher, J. M., \& Woods, J. H. (1991). MK801 and related compounds in monkeys: Discriminative stimulus effects and effects on a conditional discrimination. Journal of Pharmacology \& Experimental Therapeutics, 257, 727-734.

GilberT, M. E., \& MACK, C. M. (1990). The NMDA antagonist, MK801 , suppresses long-term potentiation, kindling, and kindlinginduced potentiation in the perforant path of the unanesthetized rat. Brain Research, 519, 89-96.

Hagen, J. J., Salamone, J. D., Simpson, J., Iversen, S. D., \& Morris, R. G. M. (1988). Place navigation in rats is impaired by lesions of medial septum and diagonal band but not nucleus basalis magnocellularis. Behavioural Brain Research, 27, 9-20.

Halliwell, R. F., \& MorRIs, R. G. M. (1987). Anticonvulsant doses of MK-801 fail to block long-term potentiation in vivo, or impair spatial memory in the rat. Neuroscience Letters, 29(Suppl.), S99.

JACKSON, A., KoeK, W., \& COLPAERT, F. C. (1992). NMDA antagonists make learning and recall state-dependent. Behavioural Pharmacology, 3, 415.421.
KEITH, J. R., \& MCVETY, K. M. (1988). Latent place learning in a novel environment and the influences of prior training in rats. Psychobiology, 16, 146-151.

KEITH, J. R., \& RudY, J. W. (1990). Why NMDA-receptor-dependent long-term potentiation may not be a mechanism of learning and memory: Reappraisal of the NMDA-receptor blockade strategy. Psychobiology, 18, 251-257.

Konarska, M., Stewart, R. E., \& McCarty, R. (1990). Habituation and sensitization of plasma catecholamine responses to chronic intermittent stress: Effects of stressor intensity. Physiology \& Behavior, 47, 647-652.

LEUNG, L. S., \& SHEN, B. (1993). Long-term potentiation in CAl: Effects of afterdischarges, NMDA antagonists, and anticonvulsants. Experimental Neurology, 119, 205-214.

MABRY, T. R., Gold, P. E., \& MCCARTY, R. (1995). Age-related changes in plasma catecholamine responses to acute swim stress. Neurobiology of Learning \& Memory, 63, 260-268.

McLamb, R. L., Williams, L. R., NanRY, K. P., Wilson, W. A., \& TrLson, H. A. (1990). MK-801 impedes the acquisition of a spatial memory task in rats. Pharmacology, Biochemistry \& Behavior, 37, $41-45$.

McNamara, R. K., de Pape, G. E., \& Skelton, R. W. (1993). Differential effects of benzodiazepine receptor agonists on hippocampal long-term potentiation and spatial learning in the Morris water maze. Brain Research, 626, 63-70.

McNamara, R. K., \& Skelton, R. W. (1991). Diazepam impairs acquisition but not performance in the Morris water maze. Pharmacology, Biochemistry \& Behavior, 38, 651-658.

MCNamaRa, R. K., \& Skelton, R. W. (1992). The neuropharmacological and neurochemical basis of place learning in the Morris water maze. Brain Research Reviews, 18, 33-49.

MCNaughton, N., \& Morris, R. G. M. (1987). Chlordiazepoxide, an anxiolytic benzodiazepine, impairs place navigation in rats. Behavioural Brain Research, 24, 39-46.

Miller, J. A., \& Richter, J. A. (1985). Effects of anticonvulsants in vivo on high affinity choline uptake in vitro in mouse hippocampal synaptosomes. British Journal of Pharmacology, 84, 19-25.

MoRRIS, R. G. M. (1981). Spatial localization does not require the presence of local cues. Learning \& Motivation, 12, 317-338.

Morris, R. G. M., ANderson, E., LyNCh, G. S., \& Baudry, M. (1986). Selective impairment of learning and blockade of long-term potentiation by an $N$-methyl-D-aspartate receptor antagonist, AP5. Nature, 319, 774-776.

Perone, M. (1991). Experimental design in the analysis of free-operant behavior. In I. H. Iverson \& K. A. Lattal (Eds.), Experimental analysis of behavior: Part 1 (pp. 135-172). Amsterdam: Elsevier.

Richter, J. A., Gormley, J. M., Holtman, J. R., \& Simon, J. R. (1982). High-affinity choline uptake in the hippocampus: Its relationship to the physiological state produced by administration of barbiturates and other treatments. Journal of Neurochemistry, 39, 1440-1445.

Robinson, G. B., \& ReED, G. D. (1992). Effect of MK-801 on the induction and subsequent decay of long-term potentiation in the unanesthetized rabbit hippocampal dentate gyrus. Brain Research, $569,78-85$.

Robinson, G. S., Jr., Crooks, G. B., Jr., Shinkman, P. G., \& GalLAGHER, M. (1989). Behavioral effects of MK- 801 mimic deficits associated with hippocampal damage. Psychobiology, 17, 156-164.

SAUCIER, D., \& CAIN, D. P. (1995). Spatial learning without NMDA receptor-dependent long-term potentiation. Nature, 378, 186-189.

Saucier, D., Hargreaves, E. L., Boon, F., Vanderwolf, C. H., \& CAIN, D. P. (1996). Detailed behavior analysis of water maze acquisition under systemic NMDA or muscarinic antagonism: Nonspatial pretraining eliminates spatial learning deficits. Behavioral Neuroscience, 110, 103-116.

SHAPIRO, M. L., \& O'CONNOR, C. (1992). N-methyl-D-aspartate receptor antagonist MK-801 and spatial memory representation: Working memory is impaired in an unfamiliar environment but not in a familiar environment. Behavioral Neuroscience, 106, 604-612.

Thompson, D. M. (1978). Stimulus control and drug effects. In 
D. Blackman \& D. Sanger (Eds.), Contemporary research in behavioral pharmacology (pp. 159-207). New York: Plenum.

ThOMPSON, D. M., \& MoERSCHBAeCHeR, J. M. (1979). Drug effects on repeated acquisition. In T. Thompson \& P. B. Dews (Eds.), Advances in behavioral pharmacology (Vol. 2, pp. 229-259). New York: Academic Press.

WHISHAW, I. Q. (1985a). Cholinergic receptor blockade in the rat impairs locale but not taxon strategies for place navigation in a swimming pool. Behavioral Neuroscience, 99, 979-1005.

WhISHAW, I. Q. (1985b). Formation of a place learning-set by the rat:
A new paradigm for neurobehavioral studies. Physiology \& Behavior, 35, 139-143.

WHISHAW, I. Q., \& AUER, R. (1989). Immediate and long-lasting effects of MK-801 on motor activity, spatial navigation in a swimming pool and EEG in the rat. Psychopharmacology, 98, 500-507.

(Manuscript received September 27, 1996; revision accepted for publication January 21,1997 .) 